\title{
A new approximate model of tyre accounting for both de- formed state and dry friction forces in the contact spot on the background of the coupled model
}

\author{
Alexey A. Kireenkov ${ }^{1, *}$, Dmitry V. Nushtaev ${ }^{2, * *}$, and Sergey I. Zhavoronok ${ }^{3, * * *}$ \\ ${ }^{1}$ Moscow Institute of Physics and Technology (State University) \\ Institute for Problems in Mechanics of Russian Academy of Sciences \\ ${ }^{2}$ TESIS Ltd. \\ ${ }^{3}$ Institute for Applied Mechanics of Russian Academy of Sciences
}

\begin{abstract}
A new approximate model of the tire rolling accounting for coupled longitudinal and lateral sliding as well as the spinning and the deformed state resulting in elastic forces is proposed. The main goal of this investigation consists in the construction of simple models with a few of degrees of freedom allowing one to implement them analytically in the engineering practice, primarily for the estimation of the rolling stability and for the prognosis of the instable rolling so-called "shimmy phenomenon" and to numerical simulation of the transient dynamics of rolling wheels with sliding effects. Such a model could become useful at the earlier stages of the engineering design instead of complex numerical models that are usually resource consuming.

The known models of the shimmy phenomenon are usually based on the nonholonomic condition of the steady rolling and consider as the main cause of instability only the tire deformation while the sliding and spinning are assumed to vanish, i. e. the dry friction effects are neglected. Another type of models consists in the "rigid wheel" assumption, in other words only the dry friction effects are accounted on the background of the coupled dry friction theory whereas the deformed state effects are neglected. Such a theory is based on the complete accounting of the combined kinematics due to simultaneous sliding and spin and shows its efficiency for slightly deformed wheels and the unsteady rolling regimes. Our goal consists in the formulation of the combined model that takes into account as well as the deformed state as the dry friction effects. This model is based on the solution of some model problems for the tire using solid finite element simulation or various shell theories, the computing of a set of specific generalized rigidity factors for the tire model, and on the accounting for the shape of the contact spot and the contact pressure distribution after the numerical simulation of the tire-road contact interaction in quasi-statics. As a result, we obtain a model with only a few degrees of freedom but more general that the Keldysh's or Klimov's ones.
\end{abstract}

\footnotetext{
*e-mail: kireenk@ipmnet.ru

**e-mail: nyshtaev.vfb@rambler.ru

***e-mail: Zhavoronok@iam.ras.ru
} 


\section{Introduction}

The dynamics of various vehicles equipped by pneumatic wheels becomes a result of many complex interactions of their structural components, but "...a major role is played by the pneumatic tyre" ([1], p. v). H. B. Paceika noted that "the mechanical behavior of the tyre needs to be investigated ...in terms of its reaction to various inputs associated with wheel motions and road conditions" [1]. The complex behavior of tires required appropriate simplified models that could be useable in the engineering practice; a wide range of so-called empirical and semi-empirical models can be mentioned, e. g. the well-known "Magic Formula Model" $[1,2]$. A more detailed description of the tire-road interaction can be offered by the Tire Brush Model [1]; an almost full survey of existing models used for pneumatic wheels was presented recently in [3]. Another way allowed by the powerful modern hard- and software consists in the direct finite element simulation of the rolling tires [4]; such an approach can be interpreted as the must detailed one, but the three-dimensional finite element models of tires are resource consumptive even in statics. This method deals primarily with final design stages and has almost nothing to do with preliminary ones where simple models with a few degrees of freedom are needed.

One of the problems of tire modeling that have intensively studied last years consists in the improved modeling of friction in the tire-road contact spot [5]. Most of the complex physical as well as empirical tire models account for the dry friction, including viscosity effects and variable boundary of sliding and adhesion zones (e. g. see [3]). It is clear that the friction acts as a main cause of wear and outage for wheels; but this is not the only friction effect. It should be noted that the dry friction has significant effects on the dynamics of rolling wheels, moreover it may cause the well-known shimmy phenomenon as it was shown in $[6,7]$. This phenomenon was found theoretically only after the introduction of the so-called Multi-Component Dry Friction theory $[8,9]$ accounting for the strong coupling of dry friction forces and torque in the case of the combined sliding and spin that was observed by Th. Erismann [10] and P. Contensou [11]. Indeed, the use of the traditional AmontonCoulomb dry friction law for vanishing contact spots leads to the results being inconsistent with the reality $[9,11]$. At the same time its incremental representation for the point of the finite contact spot with the summary sliding velocity due to the simultaneous slip and spin and further computing of resulting dry friction vector and couple by means of integration over the contact spot results in qualitatively different models that are able to predict some known effects not described by uncoupled models $[8,9]$, e. g. the shimmy of quasi-rigid wheels with negligible elastic deformations $[6,7]$. Such an effect cannot be found using the traditional shimmy theories such as the ones of M. V. Keldysh [12] and others [13] based on the non-holonomic rolling condition and neglecting the slip and spinning. The rigid wheel model has shown its efficiency in the study of the shimmy oscillations of landing gears of aircrafts observed shortly after touchdowns [14]; its further improvement requires accounting for the elastic deformations of tires to be consistent with the classical approach [1, 12]. Accounting for the real distribution of the contact pressure at different vertical loads obtained from the finite element solution [15] together with the simple approximations for resulting vectors and couple of friction [16, 17] became the first improvement of the friction-based shimmy model [18] whereas the accounting for the tire tread effect on the background of the anisotropic theory of the combined dry friction became the second one [19-21]. Here the friction anisotropy and the complex distribution of the contact pressure as well as the lateral and torsional compliance of the tire obtained from the three-dimensional finite element simulations are introduced, and the first attempt to combine the approaches [12] and [6] under a unified formulation is made. 


\section{On the anisotropic dry friction under the conditions of the combined kinematics}

\subsection{A differential formulation of the Coulomb dry friction law}

Let us consider a solid with a deformed configuration $G$ interacting with a rigid rough plane $S_{0}$ through the finite contact area $S: \partial G \cap S_{0}$, and let us assume the boundary surface $\partial G$ be smooth and convex. Let the relative motion of $G$ and $S_{0}$ be defined by the tangent velocity vector $\mathbf{v}$ defined at each point $M \in S$. Thus, for the frictional anisotropy the Coulomb dry friction law can be represented using the following local formulation $[18,19]$ :

$$
\mathbf{F}=-|p| \mathbf{f} \cdot \mathbf{v} /|\mathbf{v}| \quad|\mathbf{v}| \neq 0 ; \quad \mathbf{f}=f^{i j} \mathbf{r}_{i} \mathbf{r}_{j}
$$

Here the second rank tensor $\mathbf{f}$ is the dry friction coefficient; $\mathbf{r}_{i}$ are base vectors of some frame $x^{i}$ on the plane of interaction of the contacting bodies, $\mathbf{v}$ is the vector of the relative sliding velocity, and the symbol "." denotes the scalar product.

\section{Numerical examples}

The finite-element based numerical simulation was performed for a typical tire to obtain the global friction factors and elastic constants for the reduced model presented above. The dependence of the lateral force $F_{v}$ on the lateral displacement $\lambda$ is presented on the figure 1 while the analogous dependence of the torque on the figure 2. As it can be seen, these dependencies have almost linear initial curve pieces and almost constant second pieces. Thus, at the first stages the elastic strain exists with no sliding and spin whereas after reaching threshold displacement value $\lambda_{*}$ and $\phi_{*}$ the sliding and spin appear, and the generalized forces are dry friction forces. On the other hand, the dependencies could be easily approximated:

$$
\begin{gathered}
F(\lambda)=C_{F} \lambda, \quad \lambda \in\left[0, \lambda_{*}\right] ; \quad M(\phi)=C_{M} \phi, \quad \phi \in\left[0, \phi_{*}\right] ; \\
F(\phi)=F_{0}, \quad \lambda>\lambda_{*} . \quad M(\phi)=M_{0}, \quad \phi>\phi_{*} .
\end{gathered}
$$

The factors values as well as confidence bounds and relative mean-square errors of approximation are shown in table 1.

Table 1. Piecewise-Linear approximation for the dependence of the lateral force $F$ and torque $M_{z}$ on the lateral displacement $\lambda$ and spin angle $\phi$

\begin{tabular}{|l|c|c|}
\hline Constant & Conf. bounds & RMSE \\
\hline$C_{F}=8.274 \times 10^{4} \mathrm{~N} / \mathrm{m}$ & {$[8.2558 .299] \times 10^{4}$} & 4.534 \\
$F_{0}=1.862 \times 10^{3} \mathrm{~N}$ & {$[1.8611 .864] \times 10^{3}$} & 2.152 \\
\hline$C_{M}=1.561 \times 10^{6} \mathrm{~N}$ & {$[1.4671 .655] \times 10^{6}$} & 5302 \\
$M_{0}=8.110 \times 10^{4} \mathrm{~N} \times \mathrm{rad}$ & {$[8.1048 .116] \times 10^{4}$} & 431.3 \\
\hline
\end{tabular}

These investigations were carried out within the framework of the state program of the scientific research works on the section No.: AAAA-A17-117021310382-5 and were supported by the Moscow Institute of Physics and Technology (State University) 


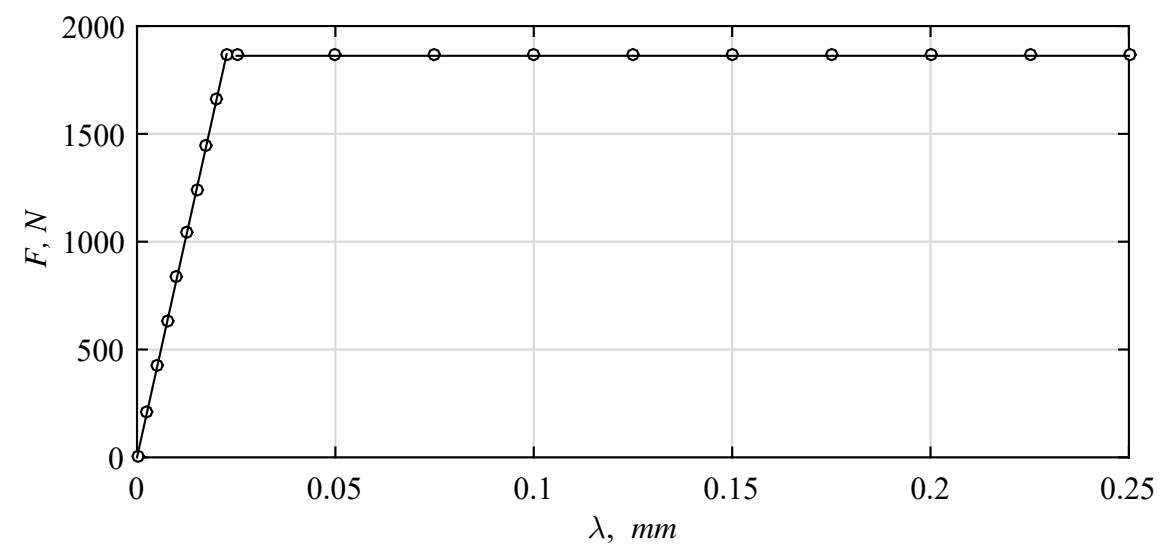

Figure 1. Dependency of the lateral force $M_{z}$ on the lateral displacement $\lambda$ : finite element simulation (dots), linear approximation (line)

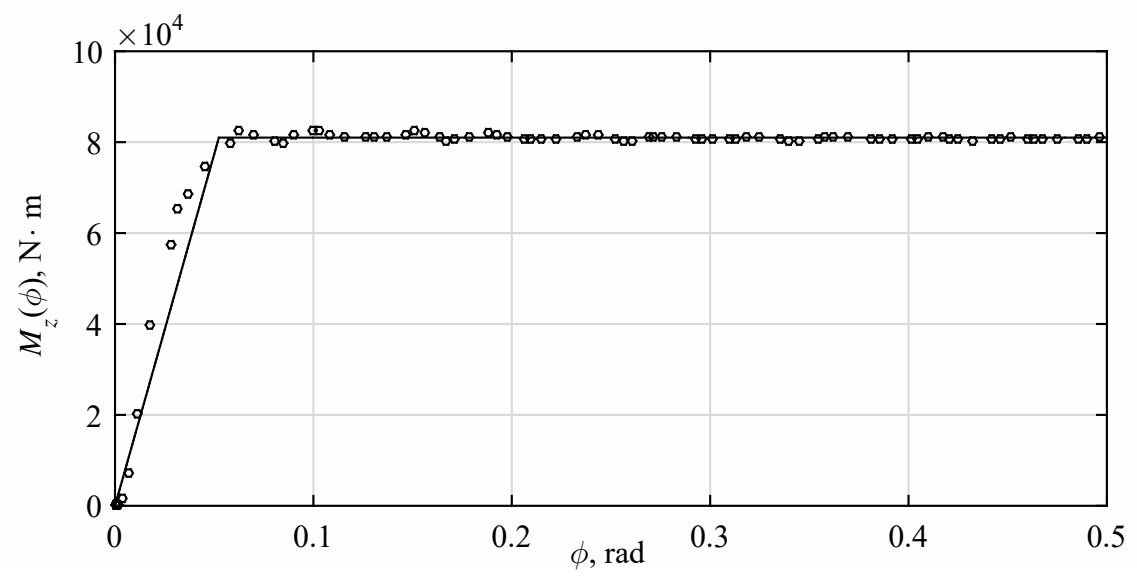

Figure 2. Dependency of the torque $M_{z}$ on the spin angle $\phi$ : finite element simulation (dots), linear approximation (line)

\section{References}

[1] H. B. Pacejka, Tyre and Vehicle Dynamics. Butterworth-Heinemann, Oxford, 2002.

[2] E. Bakker, L. Nyborg, and H. B. Paceika, SAE Paper No. 870421, 1987.

[3] R. Kiébré, Contribution to the modelling of aircraft tyre-road interaction, $\mathrm{Ph}$. D. Thesis, Univ. de Haute-Alsace, Dec. 2010.

[4] M. H. Reza Ghoreishy, Iranian Polymer Journal, 17(8), 591-597, 2017.

[5] R. van der Steen, Tyre/Road friction modeling: Literature survey. Eindhoven Univ. of Technology, Dept. of Mech. Engineering, Dynamics and Control group. Eindhoven, 2007.

[6] V. Ph. Zhuravlev and D. M. Klimov, Doklady Physics, 428(6), 761-764, 2009. 
[7] V. Ph. Zhuravlev and D. M. Klimov, Mechanics of Solids, 45(3), 324-330, 2010.

[8] V. Ph. Zhuravlev, Mechanics of Solids, 38(4), 52-58, 2003.

[9] V. V. Andronov and V. Ph. Zhuravlev, Dry Friction in Problems of Dynamics (in Russian). Regular and Chaotic Dynamics, Moscow, Izhevsk, 2010.

[10] Th. Erismann, Z. Angew. Math. Phys. 5, 355-388, 1954.

[11] P. Contensou, in: Kreiselprobleme Gydrodynamics: IUTAM Symposium, Celerina, Springer, Berlin, 201-216, 1963.

[12] M. V. Keldysh, in: Selected Works, Mechanics. Nauka, Moscow, 1985.

[13] V. S. Gozdek, Soviet Phys. Dokl. 536(14), 1969.

[14] A. A. Zagordan and S. I. Zhavoronok, Nelineiny Mir, 10, 646-656, 2011.

[15] S. E. Bogoslovskii and N. N. Kurdyumov, Proc TSU Tech Sci, 8(2), 138--147, 2015.

[16] V. Ph. Zhuravlev and A. A. Kireenkov, Mechanics of Solids, 40(2):1-10, 2005.

[17] A. A. Kireenkov, in: ENOC-2005, Eindhoven, Netherlands, 2010.

[18] A. A. Kireenkov and S. I. Zhavoronok, International Jounal of Mechanical Sciences, 127, 198-203, 2017.

[19] A. A. Kireenkov and S. I. Zhavoronok, in: Proceedings of the VII International Conference on Computational Methods for Coupled Problems in Science and Engineering: COUPLED PROBLEMS 2017, June 11-14, Rodos, Greece, 216-226, 2017.

[20] A. A. Kireenkov and S. I. Zhavoronok, in: Proceedings of 9th European Nonlinear Dynamics Conference (ENOC2017), edited by Gábor Stépán, Gábor Csernák, June 2530, 2017, Budapest, ISBN: 978-963-12-9168-1, ID 465.

[21] A. A. Kireenkov and S. I. Zhavoronok, in: Proceedings of 9th European Nonlinear Dynamics Conference (ENOC2017), edited by Gábor Stépán, Gábor Csernák, June 2530, 2017, Budapest, ISBN: 978-963-12-9168-1, ID 473. 